\title{
Achievements of Schistosomiasis Control in China
}

\author{
Hongchang Yuan ${ }^{+}$, Qingwu Jiang, Genming Zhao, Na He \\ Department of Epidemiology, School of Public Health, Fudan University, Shanghai, 200032, China
}

The control of schistosomiasis has been spectacularly successful in terms of controlling endemicity and severity of the disease during the last 50 years. It can be categorized into two stages. From 1955 through 1980, the transmission-control strategy had been widely and successfully carried out. By the end of 1980, the epidemic of schistosomiasis was successfully circumscribed in certain core regions including areas at the middle and low reaches of the Yangtze River and some mountainous areas in Sichuan and Yunnan provinces, where control of schistosomiasis had been demonstrated to be very difficult to be sustained. Therefore, since 1980, schistosomiasis control in China has been modified to employ a stepwise strategy, based on which morbidity control has been given priorities and if possible transmission control has been pursued. However, since snail-ridden areas remain unchanged so far, reinfections occur frequently. This necessitates a maintenance phase to consolidate the achievements in the control of schistosomiasis. In the mean time, we are challenged with some environmental, social and economical changes in terms of controlling schistosomiasis. Successfully controlling schistosomiasis in China is still a long-term task but will be achieved without doubt along with the economic development and the promotion of living and cultural standard of people.

Key words: control - schistosomiasis - China

Schistosomiasis in China has a long history and had been severely endemic. Since the founding of People's Republic of China in 1949, a large amount of national, provincial and local anti-schistosomiasis centers had been set up, which had carried out many large-scale surveys and massive control programs on schistosomiasis. Due to their dedication and tremendous efforts during the last 50 years, schistosomiasis has been successfully controlled or even eradicated in many areas. The present article is intended to review the epidemic and control of schistosomiasis in China during last five decades, to discuss the new challenges in the control of schistosomiasis, and to demonstrate the necessity to reconsider those previously used and effective control strategies. Some suggestions are presented as well.

\section{CONTROL EVOLUTION}

From 1955 through 1980s, comprehensive control measures targeted on schistosomiasis had been carried out with the emphasis on environmental management that was aiming at the interruption of transmission of infection. Great success was achieved in four out of twelve endemic provinces and two-thirds of the endemic counties. Endemic areas of chistosomiasis were then circumscribed in certain core regions that have been demonstrated to be much more difficult to sustain control, such as the middle and low reaches of the Yangtze River as well as some mountainous areas in Sichuan and Yunnan provinces. Consequently, a stepwise control strategy was employed for controlling schistosomiasis in these core regions. The

${ }^{+}$Corresponding author. Fax: +86-21-6403.7350. E-mail: hcyuan@shmu.edu.cn

Received 18 June 2002

Accepted 15 August 2002 first step was to control morbidity through mass chemotherapy in the severely endemic areas and selective chemotherapy in less severely endemic areas. Further steps included snail control through mollusciciding and/or environmental modification, as well as health education and sanitation. For example, in 1992 a joint project supported by the World Bank, which was also an important component of National Schistosomiasis Control Program was initiated. The primary objectives were to control morbidity due to schistosomiasis and in some areas to interrupt the transmission, if possible (Yuan et al. 2000).

The epidemic status of schistosomiasis in China has changed dramatically. When the first national control project was initiated, there were about 13 million people estimated to be infected and about 100 million people at risk. But now, schistosomiasis has been successfully controlled in 5 out of 12 former endemic provinces and in $73.8 \%$ of endemic counties (originally 413 counties). The area of snail-ridden regions was reduced by $76.6 \%$ (originally 148 billion $\mathrm{m}^{2}$ ). In the mean time, the number of infected individuals and infected cattle/buffaloes declined by $93.8 \%$ and $54.8 \%$, respectively (Chen et al. 2001). The number of patients with Katayama fever has declined year by year since 1989. Even in 1998 when the middle and low reaches of the Yangtze River, also known as ineffectively controlled endemic areas of schistosomiasis, were challenged by the biggest flooding in China in the twentieth century, no significant increase in the number of patients with Katayama fever was observed (Fig. 1).

The major characteristics of control measures of schistosomiasis in china are as the following: (1) the Chinese Communist Party and Chinese Government have been deeply concerned with the people's suffering from schistosomiasis and have provided tremendous political and financial support for schistosomiasis control; (2) several ministries and national committees such as the Ministry of Agriculture, the Ministry of Water Conservation have 


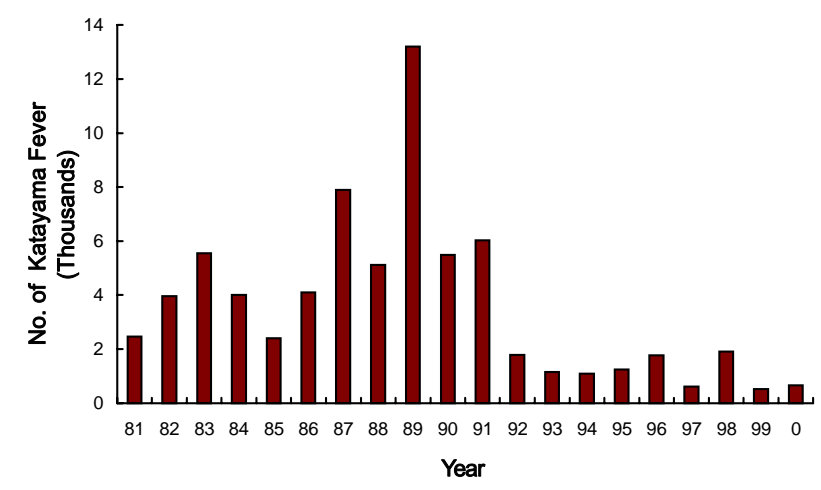

Fig. 1: the number of patients with Katayama Fever (1981-2000)

worked closely with the Ministry of Health, leading many integrated and cooperative control programs; (3) a huge amount of citizens have been motivated and actively participated in controlling schistosomiasis; (4) comprehensive control measures including active prevention and treatment have been implemented; (5) great resources and efforts have been spent in scientific research, making the control measures more cost-effective; (6) both professional and part-time personnel have played important roles in controlling schistosomiasis; (7) financial support has been greatly and successfully raised from various sources.

\section{ECOLOGICAL CHARACTERIZATIONS}

Right now, $95.7 \%$ of snail-ridden areas and $85.6 \%$ of infected individuals are located or living in the marshland or lake regions. The remaining $4.3 \%$ of the snail-ridden areas and $14.4 \%$ of infected individuals are in mountainous regions. The reason that schistosomiasis could not be well controlled around the lake and mountainous regions is related to the ecological characteristics of those areas ridden with snails and the epidemiological features of schistosomiasis over there. Socioeconomic factors have also exerted impacts on the transmission of schistosomiasis.

Marshland and lake regions - There is an apparent seasonal fluctuation of the water level of the Yangtze River and its surrounding lakes, which usually dries in Winter and floods in Summer. The river-lake terrain is overgrown with weeds, and in some areas, large sheet of reeds and willow trees are planted, which are suitable for snail breeding. Inside the embankment, where irrigation cannels are very well developed, the environment is similar to that of the plain with developed water-net in the delta of the Yangtze River in terms of the ecology of snails. It has been evident that infected cattle and buffaloes have played very important roles in the transmission of schistosomiasis in marshland and lake regions. Domestic animals are the major reservoir for schistosomiasis in lake regions and thus have been the key concern within the framework of schistosomiasis control in China (Yuan et al. 1995). The lake regions are also highly populated and the main source of marketable grain in China.

Mountainous regions - The mountainous regions refers to the high and steep mountainous areas in Yunnan and Sichuan Provinces in Southwest China. With mild climate and plentiful rainfall, snail breeds on the vertical slope. Sometimes, the endemic area and the non-endemic area are so close that they are only separated by a little hill. In the plateau canyon areas, cattle are the major reservoir for schistosomiasis. On the contrary, in the plateau basin areas, the human beings are the major reservoir. Poor minorities sparsely inhabit the vast mountainous frontier areas

\section{STRATEGY ADAPTATION}

The goal of schistosomiasis control in China is to interrupt the transmission in all amenable areas. As the endemic areas have been circumscribed in certain core regions where interruption of schistosomiasis seems to be a long-term task, a stepwise control strategy has been recommended and employed. The first step is to control morbidity supplemented with environmental modification in snail-ridden areas. The second step is to interrupt the transmission. Since 1987, morbidity control projects intended to reduce infection rates and intensities among local residents and livestock had been inaugurated in Hunan, Hubei, Jiangxi and Anhui provinces. In 1992 a World Bank supported project which was an important component of the National Schistosomiasis control Program was initiated. The objective was to reduce the prevalence of disease among human beings and cattle/buffaloes by $40 \%$, and to reduce the density of infected snails by $50 \%$. The approaches included mass chemotherapy in severely endemic areas and selective chemotherapy in moderately or mildly endemic areas, both supplemented with snail control through mollusciciding and/or environmental modification, whichever was possible. In the latest phase of this project, environmental modification was strengthened.

Two nationwide sampling surveys on schistosomiasis were conducted in 1989 and 1995, respectively (DEDC 1993, 1998). The data clearly show that the prevalence of schistosomiasis had been reduced by $52.2 \%$ (10.2\% in 1989 vs $4.89 \%$ in 1995 ) in human beings and by $31.8 \%$ (13.3\% in 1989 vs $9.1 \%$ in 1995) among cattle and buffaloes. The reduction of the prevalence was more significant in women than in man. It was also more significant in children and adolescence than in adult (Figs 2-3). In the mean time, the proportion of patients with symptoms and signs was also significantly reduced. It was also noted that the liver fibrosis at less serious extent could be reverted after several years' mass chemotherapy with praziquantel, strongly suggesting the achievement in controlling the morbidity due to schistosomiasis. This has not only been very helpful in health promotion of women, children and adolescences but also very helpful in laborforce protection.

\section{NEW CHALLENGES}

At present stage of schistosomiasis control, we are challenged with reinfection, infection in migrant population, urban schistosomiasis and the impact of water conservation projects.

Reinfection - Although the morbidity control measures have rapidly reduced the prevalence and intensity of infection, they could not interrupt the disease transmis- 


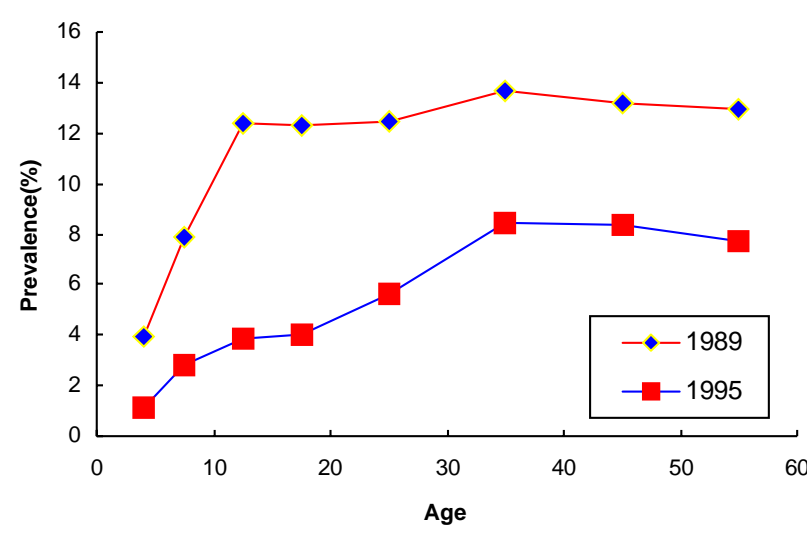

Fig 2: comparison of age-specific prevalence between 1989 and 1995 (male).

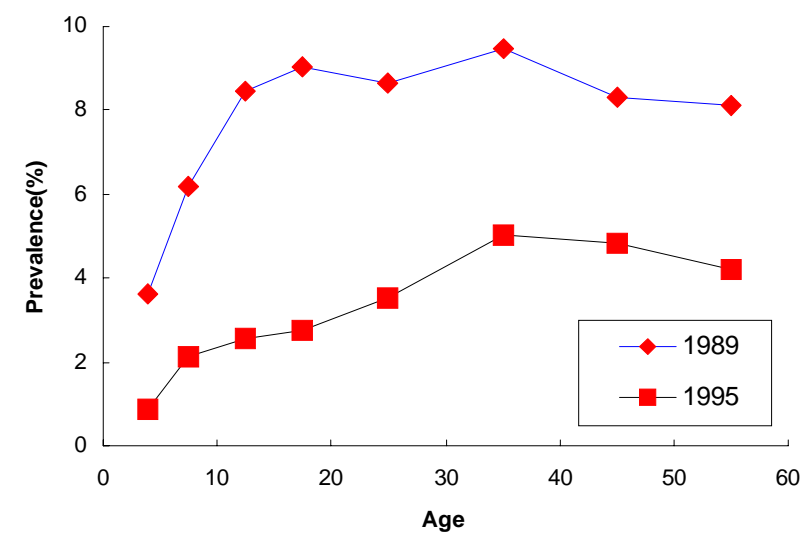

Fig 3: comparison of age-specific prevalence between 1989 and 1995 (female).

sion. Once the chemotherapeutic measure is ceased or even just its coverage rate declines, the prevalence will re-increase promptly. Villages with a high transmission rate will relapse faster than those with a low transmission rate. The fact that schistosomiasis is really a zoonosis in China renders its control to be a complex and hard task.

Migration of population - The patterns of migration can have significant impact on the transmission and control of schistosomiasis. Migrants or travelers who moved from non-endemic areas into endemic areas usually lack resistance to schistosomiasis. They should pay attention to prevention from being infected with schistosomiasis. On the other hand, there have been a huge amount of people migrating from rural endemic areas to much developed urban areas in the East and Southeast China where schistosomiasis have been successfully controlled. This can make the disease surveillance a difficult and tough task provided that snails exist somewhere and sometimes in these areas.

Urban schistosomiasis - During the summer of 1989 an outbreak of Katayama fever occurred in a district of Wuhan, the capital of Hubei province, which was previously a non-endemic area of schistosomiasis. The epidemiological investigation showed that some swamp sites near the river embankment had been ridden with snails spread from the upper steam and were then contaminated by stools of migrants from endemic areas. After the outbreak the snail breeding areas were thoroughly modified, and schistosomiasis infections have no longer occurred in this areas. Based on our study results, we should highlight the importance of identifying snail breeding areas as well as the patterns of transmission. It is also necessary to conduct surveys on schistosomiasis among immigrants who settle down near the snail breeding areas.

Impact of the Three Gorges Dam project on schistosomiasis - There is no doubt that the Three Gorges Dam which is currently being constructed will make great contributions to the economic development of China. However, changes of the ecological system would potentially influence the transmission of schistosomiasis, a potential new threat for schistosomiasis control in China. Efforts should be made as soon as possible in order to prevent the threat to be a true problem. According to our knowledge and experience, water conservation project usually has two different impacts on the endemic of schistosomiasis: either positive or negative. In China there are many examples in which schistosomiasis has been eradicated due to construction of man-made lakes in those endemic areas. On the other hand, there are also examples in which schistosomiasis has been introduced or prevailed due to construction of man-made lakes or reservoirs. We strongly suggest that a surveillance system targeting on the possibility and the pattern of schistosomiasis transmission be well organized and conducted in such kind of areas. Snail breeding areas should be eliminated before the initiation of the construction work, i.e. interventions should be taken from the beginning of the construction.

In conclusion, ongoing efforts over the last 50 years aiming at the eradication of schistosomiasis in China have made great achievements in the disease control. Nevertheless, a maintenance phase is necessary in terms of consolidating the achievement in schistosomiasis control. With the deep concern of Chinese government about people's suffering from schistosomiasis and with social and economic development, schistosomiasis will be successfully controlled in China.

\section{REFERENCES}

Chen XY, Jiang QW, Zhao GM 2001. Communication of endemic status of schistosomiasis in China in 2000. Chin J Schist Control 13: 129-131.

DEDC-Department of Endemic Diseases Control, Ministry of Health, the People's Republic of China 1993. Epidemiological Situation of Schistosomiasis in China. Results from a Nationwide Sampling Survey in 1989, Press of Chengdu Science and Technology University, China, p.16-30.

DEDC-Department of Endemic Diseases Control, Ministry of Health, the People's Republic of China 1998. Epidemic Status of Schistosomiasis in China: a Nationwide Sampling Survey in 1995, Press of Nanjing University, Nanjing, 1728 pp.

Yuan HC, Guo JG, Bergquist R 2000. The 1992-1999 World Bank schistosomiasis research initiative in China: outcome and perspectives. Parasitol Intern 49: 95-207.

Yuan HC, Zhou SJ, Zhang SJ 1995. Study on epidemiology and control strategies for control of Schistosomiasis japonica in lake and marshland regions. Chin J Schist Control 7: 193201. 
190 Schistosomiasis Control in China - Hongchang Yuan et al. 